\title{
Highly efficient yeast-based in vivo DNA cloning of multiple DNA fragments and the simultaneous construction of yeast/ Escherichia coli shuttle vectors
}

\author{
Ei'ichi lizasa and Yukio Nagano \\ Saga University, Saga, Japan \\ BioTechniques 40:79-83 (January 2006) \\ doi 10.2144/000112041
}

In vivo recombinational cloning in yeast is a very efficient method. Until now, this method has been limited to experiments with yeast vectors because most animal, insect, and bacterial vectors lack yeast replication origins. We developed a new system to apply yeast-based in vivo cloning to vectors lacking yeast replication origins. Many cloning vectors are derived from the plasmid $p B R 322$ and have a similar backbone that contains the ampicillin resistance gene and pBR322-derived replication origin for Escherichia coli. We constructed a helper plasmid $\mathrm{pSUO}$ that allows the in vivo conversion of a pBR322-derived vector to a yeast/E. coli shuttle vector through the use of this backbone sequence. The DNA fragment to be cloned is PCR-amplified with the addition of 40 bp of homology to a pBR322-derived vector. Cotransformation of linearized pSU0, the pBR322-derived vector, and a PCR-amplified $D N A$ fragment, results in the conversion of the pBR322-derived vector into a yeast/E. coli shuttle vector carrying the DNA fragment of interest. Furthermore, this method is applicable to multifragment cloning, which is useful for the creation of fusion genes. Our method provides an alternative to traditional cloning methods.

\section{INTRODUCTION}

Escherichia coli-based traditional cloning methods use restriction enzyme cleavage followed by DNA joining with DNA ligase (1). These methods are often inefficient and laborious, particularly for the cloning of multiple DNA fragments or a large DNA fragment. On the other hand, yeast cells are an ideal tool for conducting efficient in vivo recombinational cloning (2). Because most animal, insect, and bacterial vectors carry no yeast replication origins, researchers have previously been limited to using yeast-based in vivo cloning procedures for the construction of vectors in experiments involving yeast; for example, yeast two-hybrids $(3,4)$. In order to clone a desired DNA fragment into the vectors lacking yeast replication origins, the yeast-based in vivo cloning method usually requires two rounds of DNA manipulation. In the first round, the vector is converted to a yeast $/ E$. coli shuttle vector by integrating the replication origin and a selectable marker for yeast using an E. coli-based traditional cloning method. In the second round, the desired DNA fragment is PCR-amplified with $20-40$ bp of homology to a yeast vector $(2,5)$ and is cotransformed with the linearized vector into yeast.

To eliminate these two rounds of lengthy DNA manipulation, we developed a new yeast-based in vivo cloning method. Many cloning vectors are derived from the pBR322 plasmid and contain a common backbone sequence carrying an ampicillin resistance $\left(A m p^{r}\right)$ gene and a pBR322derived replication origin for $E$. coli (pBR322 origin) (6). We constructed a helper plasmid pSU0 that allows the in vivo conversion of a pBR322derived vector to a yeast/E. coli shuttle vector through the use of this backbone sequence. Cotransformation of pSU0 into yeast with a pBR322-derived vector and the desired DNA fragment results in the conversion of the pBR322-derived vector into a yeast/E. coli shuttle vector and simultaneously allows the cloning of the DNA fragment into the same vector. In addition, we show that this method is applicable to multifragment cloning (7). Our new method can replace lengthy traditional cloning methods with an efficient onestep protocol.

\section{MATERIALS AND METHODS}

\section{Strains}

E. coli strain DH5 $\alpha$ was used for the propagation of plasmids, and the strain BL21 Star TM (DE3) (Invitrogen, Carlsbad, CA, USA) was used for the expression of recombinant proteins. Yeast strain YPH499 (MATa ura3-52 lys2-801 amber ade2-101 ${ }^{\text {orchre }}$ trp 1$\Delta 63$ his3- $\Delta 200$ leu2- $\Delta 1$ ) was used for homologous recombination.

\section{Construction of pSU0}

The helper plasmid pSU0 $\left(\right.$ GenBank $^{\circledR}$ accession no. AB215109; Figure 1A) was constructed by yeast homologous recombination. This plasmid has an $A m p^{r}$ gene, a $2 \mu$ yeast replication origin $(2 \mu$ origin), a $U R A 3$ selectable marker, and a pUC-derived replication origin for $E$. coli (pUC origin) (Figure 1A). There is a single nucleotide difference between the pBR322 and pUC origins (8). First, to rearrange these origins and genes in the desired order, they were PCR-amplified from pYES2/CT (Invitrogen). For the PCR amplification of the Amp ${ }^{r}$ gene, $2 \mu$ origin- $U R A 3$, and the pUC origin, the primer pairs used were $\mathrm{pSU} 0-1$ and pSU0-4 $\left(15\right.$ cycles of $95^{\circ} \mathrm{C}$ for $30 \mathrm{~s}, 55^{\circ} \mathrm{C}$ for $30 \mathrm{~s}$, and $72^{\circ} \mathrm{C}$ for 1 min using $P f u$ DNA polymerase; Fermentas, Vilnius, Lithuania), pSU02 and pSU0-5 (40 cycles of $95^{\circ} \mathrm{C}$ for $30 \mathrm{~s}, 55^{\circ} \mathrm{C}$ for $30 \mathrm{~s}$, and $72^{\circ} \mathrm{C}$ for 3 min using $P f u$ DNA polymerase), and pSU0-3 and pSU0-6 $\left(15\right.$ cycles of $95^{\circ} \mathrm{C}$ for $30 \mathrm{~s}, 55^{\circ} \mathrm{C}$ for $30 \mathrm{~s}$, and $72^{\circ} \mathrm{C}$ for 1 min using $P f u$ DNA polymerase), respectively (Table 1 ). The primer pSU0-1 was designed to provide EcoRI and BamHI sites. Next, in order to provide the long overlapping sequence 
to these fragments, two adjacent fragments were joined by the PCRbased overlap extension method (9) as follows. Using PCR, the Amp ${ }^{r}$ and $2 \mu$ origin of replication-URA3 selectable marker fragments, $2 \mu$ origin- $U R A 3$ and the pUC origin fragments, and the pUC origin and $\mathrm{Amp}^{\mathrm{r}}$ fragments were joined by the pSU0-1 and pSU0-5, pSU0-2 and pSU0-6, and pSU0-3 and pSU0-4 primer pairs, respectively (45 cycles of $95^{\circ} \mathrm{C}$ for $30 \mathrm{~s}, 55^{\circ} \mathrm{C}$ for $30 \mathrm{~s}$, and $72^{\circ} \mathrm{C}$

A

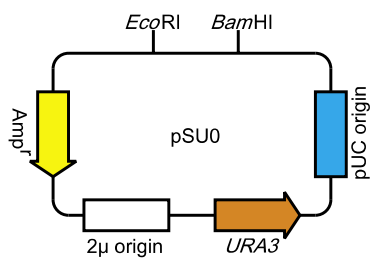

C

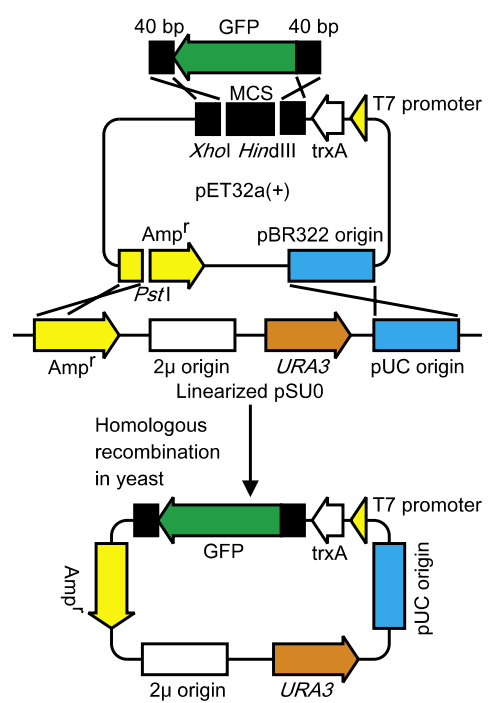

for 4 min using Pfu DNA polymerase). Cotransformation of these three DNA fragments into yeast (10) resulted in homologous recombination at the overlapping sequences and the consequent formation of the plasmid pSU0. The proper formation of pSU0 was confirmed by restriction analysis (data not shown). For the cloning experiments, the plasmid pSU0 was digested with BamHI and EcoRI and purified by electrophoresis.

B

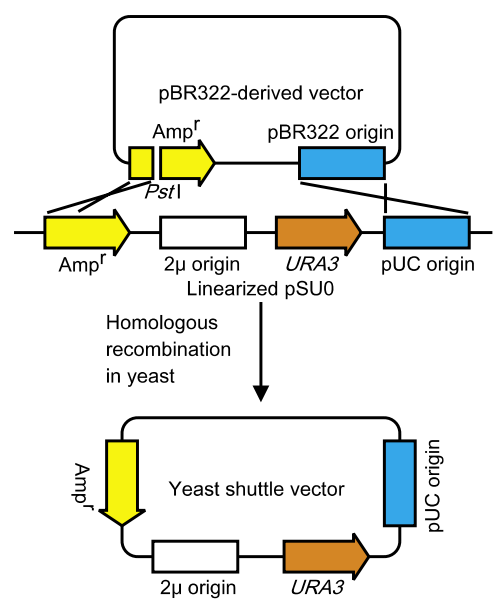

D

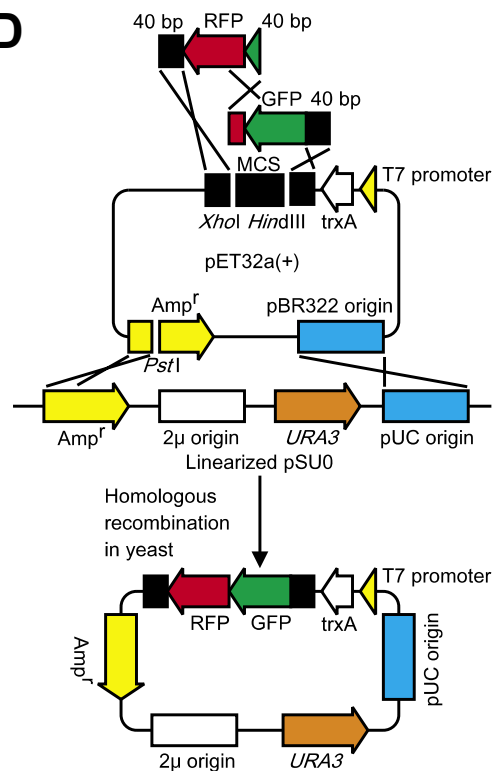

Figure 1. Diagram of the yeast-based in vivo cloning system. (A) Map of the helper plasmid pSU0. (B) The diagram of in vivo conversion of a pBR322-derived vector to a yeast/Escherichia coli shuttle vector. $(\mathrm{C}$ and $\mathrm{D})$ The diagrams of simultaneous conversion of the pET32a(+) to a yeast/E. coli shuttle vector and cloning of (C) a GFP fragment or both (D) GFP and RFP fragments into the vector. GFP, green fluorescent protein; RFP, red fluorescent protein; MCS, multiple cloning site.

\section{PCR Amplification of GFP and RFP Genes}

Open reading frames (ORFs) of green fluorescent protein (GFP) and red fluorescent protein (RFP) genes were obtained by PCR using the plasmids pBAD-GFPuv (Clontech Laboratories, Mountain View, CA, USA; accession no. U62637) and pDsRed2 (Clontech Laboratories) as DNA templates, respectively. First, for the GFP cloning experiment, the GFP gene was PCRamplified with primers pGFPF and pGFPR2 $\left(15\right.$ cycles of $98^{\circ} \mathrm{C}$ for 10 s, $55^{\circ} \mathrm{C}$ for $30 \mathrm{~s}$, and $72^{\circ} \mathrm{C}$ for $1 \mathrm{~min}$ using Pyrobest ${ }^{\circledR}$ DNA polymerase; TAKARA BIO, Ohtsu, Shiga, Japan). Subsequently, to provide this GFP gene with ends homologous to pET32a(+) (Novagen, Madison, WI, USA) (Figure 1C), the GFP fragment was PCRamplified with primers pSUOHindIII and pSUOXhoI, the $5^{\prime}$ ends of which include 40-nucleotide sequences homologous to the multiple cloning site (MCS) of pET32a(+) (35 cycles of $98^{\circ} \mathrm{C}$ for $10 \mathrm{~s}, 55^{\circ} \mathrm{C}$ for $30 \mathrm{~s}$, and $72^{\circ} \mathrm{C}$ for 1 min using Pyrobest DNA polymerase). The pET32a(+) vector is designed for expression of the target protein fused with thioredoxin under the control of a T7 promoter (11). An E. coli strain, such as BL21 Star (DE3), carries a chromosomal copy of the T7 RNA polymerase gene under the control of the lacUV5 promoter. The addition of isopropyl- $\beta$-D-thiogalactopyranoside (IPTG) to this strain induces the expression of T7 RNA polymerase followed by the expression of the thioredoxin-fused target protein.

For the multifragment cloning experiment, the fragments of the GFP and RFP genes were designed to share overlapping 40-bp sequences at their junction and to include 40-bp sequences at their nonoverlapping ends that are homologous to the MCS of pET32a(+) (Figure 1D). To obtain these fragments, the GFP gene was PCR-amplified with the primers pGFPF and pGFPR (15 cycles of $98^{\circ} \mathrm{C}$ for $10 \mathrm{~s}, 55^{\circ} \mathrm{C}$ for 30 s, and $72^{\circ} \mathrm{C}$ for 1 min using Pyrobest DNA polymerase) and subsequently with the primers pSUOHindIII and pGFPR $\left(35\right.$ cycles of $98^{\circ} \mathrm{C}$ for $10 \mathrm{~s}$, $55^{\circ} \mathrm{C}$ for $30 \mathrm{~s}$, and $72^{\circ} \mathrm{C}$ for 1 min using Pyrobest DNA polymerase). RFP was 
Table 1. Primers Used in this Study

\begin{tabular}{|ll|}
\hline Name & Sequence \\
\hline pSU0-1 & 5'-CGGGATCCATCGGAATTCATATTGAAAAAGGAAGAGTATG-3' \\
pSU0-2 & 5'-TGAAGATCCTTTTTGATAATAATCTTCCTGTTTTTGGGGC-3' \\
pSU0-3 & 5'-AATTATATCAGTTATTACCCCTCATGACCAAAATCCCTTA-3' \\
pSU0-4 & 5'-GCCCCAAAAACAGGAAGATTATTATCAAAAAGGATCTTCA-3' \\
pSU0-5 & 5'-TAAGGGATTTTGGTCATGAGGGGTAATAACTGATATAATT-3' \\
pSU0-6 & 5'-ATGAATTCCGATGGATCCCGGCGGTAATACGGTTATCCAC-3' \\
pGFPF & $5^{\prime}$-TTCGAGCTCCGTCGAGCTAGCAAAGGAGAAGAACTTTTCA-3' \\
pGFPR & 5'-ATGACGTTCTCGGAGGAGGCTTTGTAGAGCTCATCCATGC-3' \\
pGFPR2 & 5'-AGCAGCCGGATCTCATTATTTGTAGAGCTCATCCATGCCA-3' \\
pRFPF & 5'-GCATGGATGAGCTCTACAAAGCCTCCTCCGAGAACGTCAT-3' \\
pRFPR & 5'-AGCAGCCGGATCTCACTACAGGAACAGGTGGTGGCGGCCC-3' \\
pSU0HindIII & 5'-GGCCATGGCTGATATCGGATCCGAATTCGAGCTCCGTCGA-3' \\
pSU0Xhol & 5'-ACTCAGCTTCCTTTCGGGCTTTGTTAGCAGCCGGATCTCA-3' \\
\hline
\end{tabular}

also amplified with primers $\mathrm{pRFPF}$ and pRFPR $\left(15\right.$ cycles of $98^{\circ} \mathrm{C}$ for 10 $\mathrm{s}, 55^{\circ} \mathrm{C}$ for $30 \mathrm{~s}$, and $72^{\circ} \mathrm{C}$ for $1 \mathrm{~min}$ using Pyrobest DNA polymerase) and subsequently with the primers $\mathrm{pRFPF}$ and pSU0XhoI $\left(35\right.$ cycles of $98^{\circ} \mathrm{C}$ for $10 \mathrm{~s}, 55^{\circ} \mathrm{C}$ for $30 \mathrm{~s}$, and $72^{\circ} \mathrm{C}$ for 1 min using Pyrobest DNA polymerase). These PCR-amplified DNA fragments were electrophoretically purified and used for cloning into pET32a(+).

\section{Yeast and Escherichia coli Transformation}

For efficient cloning (12), the pET32a(+) vector was digested with HindIII and XhoI in its MCS and with PstI in its Amp ${ }^{\mathrm{r}}$ gene. The digested vector was not purified further. After overnight incubation of yeast cells in $5 \mathrm{~mL}$ of double-strength yeast extractpeptone-adenine-dextrose ( $2 \times$ YPAD) broth at $30^{\circ} \mathrm{C}$, they were inoculated into $50 \mathrm{~mL}$ of $2 \times$ YPAD broth and grown to obtain a cell density of $1.7 \times 10^{7}$ cells/ $\mathrm{mL}$. The yeast cells were harvested and transformed by the method of Gietz and Woods (10). However, the transformation procedure was a quarter scale of the published method. These cells were cotransformed with the linearized pSU0, digested pET32a(+), and the relevant PCR fragments. Fifty nanograms of the digested pET32a(+) correspond to 1 in the molar ratio of mixed DNA shown in Table 2. The transformants were selected using synthetic complete plates lacking uracil, incubated at $30^{\circ} \mathrm{C}$. We obtained more than 1000 transformants per 50 $\mathrm{ng}$ of the digested pET32a(+) in the GFP cloning experiment. We also obtained about 300 transformants per $50 \mathrm{ng}$ of the digested pET32a(+) in the multifragment cloning experiment. All colonies were scraped from the plates with plating beads, and the collected yeast cells were disrupted by acidwashed glass beads (425-600 $\mu \mathrm{m}$; Sigma-Aldrich, Steinheim, Germany). The plasmids were purified from the disrupted cells using a Mini- $\mathrm{M}^{\circledR}$ Plasmid DNA Extraction System (Viogene, Taipei, Taiwan). The rescued plasmids were transformed into $E$. coli DH5 $\alpha$ for propagation. The propagated plasmids were then transformed into E. coli BL21 Star (DE3), and plated on agar containing $100 \mu \mathrm{g} / \mathrm{mL}$ ampicillin and $0.01 \mathrm{mM}$ IPTG. The colonies expressing fused protein were counted.

\section{RESULTS AND DISCUSSION}

Cotransformation of the linearized helper plasmid pSU0 (Figure 1A) into yeast with the pBR322-derived vector that has been digested in the backbone sequence results in homologous recombination (Figure 1B). Using pSU0, we developed a new yeast-based in vivo cloning method that allows simultaneous conversion of a pBR322-derived vector into a yeast/E. coli shuttle vector and cloning of DNA inserts of interest by one-step transformation of yeast (Figure 1C).

To test this system, we cloned the GFP gene into an E. coli expression vector, pET32a(+). First, PCR was used to amplify the ORF of the GFP gene with $40 \mathrm{bp}$ of homology to the MCS of pET32a(+), such that homologous recombination would create an inframe fusion of GFP with thioredoxin. Subsequently, to clone the GFP into pET32a(+), yeast was cotransformed (10) with the GFP gene, the digested pET32a(+), and the linearized pSU0 (Figure 1C). In this experiment, these DNA fragments were mixed at various molar ratios (Table 2). We rescued the plasmids from all yeast transformants. The rescued plasmids were transformed into $E$. coli $\mathrm{DH} 5 \alpha$ for propagation because the protein expression host, BL21 Star (DE3), does not have high transformation efficiency. The propagated plasmids were then transformed into the BL21 Star (DE3), and colonies expressing thioredoxin-fused GFP were counted (Table 2). The difference in the ratio of DNA fragments added had little effect on the recombination frequency. Under any conditions, more than $80 \%$ of all transformants expressed thioredoxin-fused GFP. To compare this new method with the conventional yeastbased in vivo cloning method, yeast was also cotransformed with a GFP fragment and pET32a(+) carrying the yeast $2 \mu$ origin of replication and yeast selectable marker, URA3, which were mixed in a 1:1 molar ratio. Subsequent analysis of the rescued plasmids showed that $91 \%$ have the ability to express thioredoxin-fused GFP. Thus, little difference was detected in recombination efficiency between the two methods.

Restriction analysis was used to examine four colonies expressing thioredoxin-fused GFP, which were obtained by the new method. The plasmids were extracted, digested with EcoRI and DraIII, and analyzed by electrophoresis. In all lanes, 4.6, 3.6, and $1.2 \mathrm{~kb}$ fragments were present (Figure 2A, lanes 1-4). This pattern matched the predicted pattern (Figure 2B). DNA sequencing of these clones also confirmed the correct integration of the GFP gene (data not shown). 
Table 2. The Efficiencies of One-Step Yeast-Based In Vivo Cloning

\begin{tabular}{|cc|}
$\begin{array}{c}\text { Molar Ratio of } \\
\text { Mixed DNA } \\
\text { pET32a(+):pSU0:GFP }\end{array}$ & $\begin{array}{c}\text { Percentage } \\
\text { of Escherichia } \\
\text { coli Colonies } \\
\text { Expressing GFP }\end{array}$ \\
\hline $1: 1: 1$ & 88 \\
$1: 1: 6$ & 88 \\
$1: 1: 12$ & 90 \\
$1: 6: 1$ & 85 \\
$1: 12: 1$ & 88 \\
$6: 1: 1$ & 84 \\
$12: 1: 1$ & 83 \\
Each experiment was repeated three times, and \\
the averages are shown.
\end{tabular}

To determine how clones not expressing thioredoxin-fused GFP were generated, we examined plasmids from four such clones. Interestingly, some of them showed the same restriction pattern as those from colonies expressing thioredoxin-fused GFP (Figure 2A, lanes 5, 7, and 8).

DNA sequencing indicated that three clones showed frameshift mutations at the sites targeted by the PCR primers. The fourth clone not expressing thioredoxinfused GFP was created by the incorrect joining of pSU0 and the XhoI-PstI fragment of pET32a(+) by homologous recombination and nonhomologous end-joining (Figure 2A, lane 6) (13).

One of the most important advantages of yeast-based in vivo cloning is that it allows one to clone multiple PCRamplified fragments with high efficiency (7). In order to evaluate this new method, we cloned the GFP and RFP genes simultaneously into pET32a (+) (Figure 1D). We amplified the ORFs of GFP and RFP and added $40 \mathrm{bp}$ overlaps by PCR, such that homologous recombination would create in-frame fusion of thioredoxin, GFP, and RFP (Figure 1D). Because varying ratios of the DNA fragments had little effect on the recombination frequency

\section{A}

C in the GFP cloning experiment, yeast was transformed (10) with the GFP fragment, RFP fragment, the digested pET32a(+), and linearized pSU0 mixed in a $1: 1: 1: 1$ molar ratio. The rescued plasmid was amplified in DH5 $\alpha$ cells and then transformed into BL21 Star (DE3) for recombinant protein expression. The colonies expressing thioredoxin-fused GFP-RFP were counted and $65 \%$ were found to express the fusion protein.

We examined four colonies expressing thioredoxin-fused GFPRFP by restriction analysis. In all lanes, 4.6, 3.6, and $1.9 \mathrm{~kb}$ fragments were present (Figure 2C, lanes 1-4). This pattern matched the predicted pattern (Figure 2D). DNA sequencing of these clones also confirmed the correct integration of both genes (data not shown).

To determine the events responsible for the production of clones not expressing thioredoxin-fused GFPRFP, we analyzed plasmids from four colonies not expressing GFP-RFP. In one clone, a frameshift mutation occurred at the site targeted by PCR primers (Figure 2C, lane 7). Other errors included the conversion of pET32a $(+)$, which was not digested in the MCS, to a yeast/E. coli shuttle vector (Figure 2C, lane 5); cloning of an N-truncated GFP with intact RFP into the vector (Figure 2C, lane 6); and integration of unwanted pDsRed and pBAD-GFPuv sequences into pSU0 (Figure 2C, lane 8).

The main factor responsible for clones not expressing fused protein was the occurrence of frameshift mutations at the sites targeted by the PCR primers (Figure 2A, lanes 5, 7, and 8; Figure $2 \mathrm{C}$, lane 7). It is likely that errors in the synthesis of the PCR primers caused these mutations.

The new cloning method described here is highly efficient. It is applicable
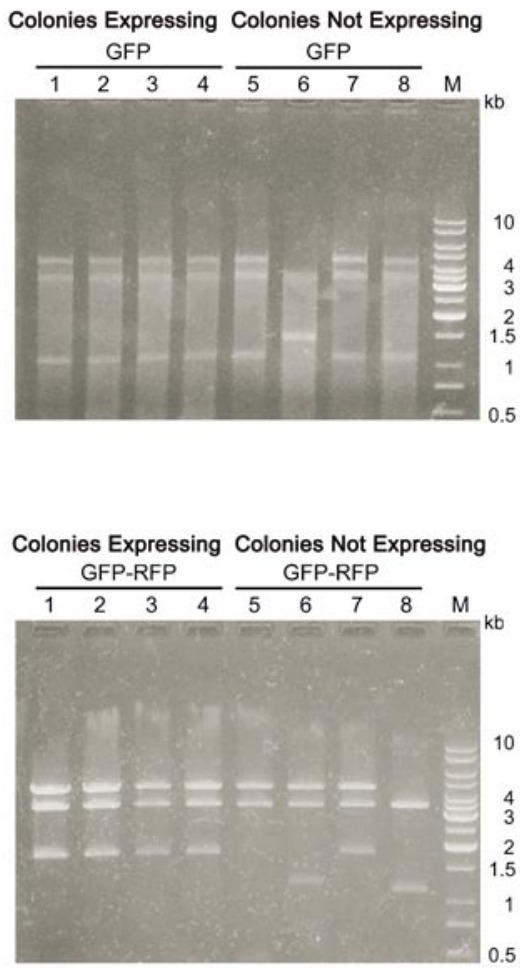

Figure 2. Restriction analysis of plasmids from transformants that were obtained in the (A and B) GFP cloning and (C and D) multifragment cloning experiments. (A and C) Plasmids from transformants were digested by DraIII and $E c o R I$ and analyzed by electrophoresis on a $1 \%$ agarose gel. The GeneRuler ${ }^{\mathrm{TM}} 1 \mathrm{~kb}$ DNA Ladder (Fermentas) is indicated on the right (M). (B) Diagram showing the restriction patterns of the pET32a $(+)$ containing the $2 \mu$ origin of replication, URA3 selectable marker, and GFP gene. (D) Diagram showing the restriction patterns of the pET32a(+) containing $2 \mu$ origin of replication, URA3 selectable marker, and both GFP and RFP genes. GFP, green fluorescent protein; RFP, red fluorescent protein. 
to multifragment cloning, allowing fusion genes to be easily obtained by a one-step transformation of yeast. This provides a clear alternative to the traditional E. coli-based cloning methods using restriction enzyme cleavage followed by DNA joining with DNA ligase.

Many E. coli-based in vivo cloning methods have been previously reported $(14,15)$. However, for efficient cloning in $E$. coli, special genotypes and electroporation apparatus are needed for transformation. In addition, efficient cloning in $E$. coli-based methods requires longer homologous sequences than those used in yeast-based methods $(14,15)$. Unlike in E. coli, there is little need for special genotypes for in vivo cloning in yeast. Most yeast strains, including those used for two-hybrid methods, have a high rate of recombination, and yeast transformation does not require an electroporation apparatus for efficient transformation. Thus, our new cloning method is readily available to researchers conducting yeast twohybrid experiments.

\section{ACKNOWLEDGMENTS}

We thank Dr. T. Anai and Dr. S. Katayama for their helpful suggestions.

\section{COMPETING INTERESTS STATEMENT}

The authors declare no competing interests.

\section{REFERENCES}

1. Cohen, S.N., A.Y. Chang, H.W. Boyer, and R.B. Helling. 1973. Construction of biologically functional bacterial plasmids in vitro. Proc. Natl. Acad. Sci. USA 70:3240-3244.

2. Oldenburg, K.R., K.T. Vo, S. Michaelis, and C. Paddon. 1997. Recombination-mediated PCR-directed plasmid construction in vivo in yeast. Nucleic Acids Res. 25:451-452.

3. Martzen, M.R., S.M. McCraith, S.L. Spinelli, F.M. Torres, S. Fields, E.J. Grayhack, and E.M. Phizicky. 1999. A biochemical genomics approach for identifying genes by the activity of their products. Science 5:1153-1155.

4. Uetz, P., L. Giot, G. Cagney, T.A. Mansfield, R.S. Judson, J.R. Knight, D. Lockshon, V. Narayan, et al. 2000. A comprehensive analysis of protein-protein interactions in Saccharomyces cerevisiae. Nature 403:623627.

5. Hua, S., M. Qiu, E. Chan, L. Zhu, and Y. Luo. 1997. Minimum length of sequence homology required for in vivo cloning by homologous recombination in yeast. Plasmid 38:91-96.

6. Bolivar, F., R.L. Rodriguez, M.C. Betlach, and H.W. Boyer. 1977. Construction and characterization of new cloning vehicles. I. Ampicillin-resistant derivatives of the plasmid pMB9. Gene 2:75-93.

7. Marykwas, D.L. and S.E. Passmore. 1995. Mapping by multifragment cloning in vivo. Proc. Natl. Acad. Sci. USA 92:11701-11705.

8. Vieira, J. and J. Messing. 1982. The pUC plasmids, an M13mp7-derived system for insertion mutagenesis and sequencing with synthetic universal primers. Gene 19:259-268.

9. Ho, S.N., H.D. Hunt, R.M. Horton, J.K. Pullen, and L.R. Pease. 1989. Site-directed mutagenesis by overlap extension using the polymerase chain reaction. Gene 77:51-59.

10.Gietz, R.D. and R.A. Woods. 2002. Transformation of yeast by lithium acetate/ single-stranded carrier DNA/polyethylene glycol method. Methods Enzymol. 350:8796.

11.LaVallie, E.R., E.A. DiBlasio, S. Kovacic, K.L. Grant, P.F. Schendel, and J.M. McCoy. 1993. A thioredoxin gene fusion expression system that circumvents inclusion body formation in the E. coli cytoplasm. Biol. Technology 11:187-193.

12.Raymond, C.K., T.A. Pownder, and S.L. Sexson. 1999. General method for plasmid construction using homologous recombination. BioTechniques 26:134-141.

13.Wilson, J.H., P.B. Berget, and J.M. Pipas. 1982. Somatic cells efficiently join unrelated DNA segments end-to-end. Mol. Cell. Biol. 2:1258-1269.

14.Zhang, Y., J.P. Muyrers, G. Testa, and A.F. Stewart. 2000. DNA cloning by homologous recombination in Escherichia coli. Nat. Biotechnol. 18:1314-1317.

15.Yu, D., H.M. Ellis, E.C. Lee, N.A. Jenkins, N.G. Copeland, and D.L. Court. 2000. An efficient recombination system for chromosome engineering in Escherichia coli. Proc. Natl. Acad. Sci. USA 97:5978-5983.

Received 21 June 2005; accepted 31 August 2005.

Address correspondence to Yukio Nagano, Analytical Research Center for Experimental Sciences, Saga University, Saga 840-8502, Japan.e-mail:nagano@cc.saga-u.ac.jp.

\footnotetext{
To purchase reprints

of this article, contact

Reprints@BioTechniques.com
} 